\title{
Tensile properties and micromorphologies of sawdust and chipwood filled epoxy composites
}

\begin{abstract}
In this study, waste wood product obtained from the timber industry originating from various type of wood has been investigated. Research work carried out on the three different sizes of fiber derived from the sawdust (SW) and chip wood (CW). The SW and CW fiber and epoxy resin were blended together respectively using hand tools machine, all specimens of fiber composite were prepared accordance to the ASTM standards. Tensile and morphological properties provide an excellent measure of the degree of reinforcement provided by the fiber to the composite. The tensile modulus increased with the filler size from coast to rough of composites both SW and CW but decreases steadily with the woodï fiber size from rough to soft content. Statistical analysis using one way and two ways analysis of variances (ANOVA) showed that the differences of results obtained from those SW and CW fiber composite samples are significant, which confirm a very firm mechanical performance of the composites through tensile tests. This shows the producing a good quality of SW and CW fibres composite which maybe used for home furniture utilities.
\end{abstract}

Keyword: Sawdust; Chip wood; Wood composites; Epoxy composites 